\title{
COMMENTARY
}

\section{Cerebrospinal fluid lactate: Is it a reliable and valid marker to distinguish between acute bacterial meningitis and aseptic meningitis?}

\author{
Kameshwar Prasad* and Jitendra Kumar Sahu \\ See related research by Huy et al., http://ccforum.com/content/14/6/R240
}

\begin{abstract}
Cerebrospinal fluid (CSF) lactate assay has been a subject of research since 1925. A systematic review by Huy and colleagues in the previous issue of Critical Care summarizes data from 25 studies evaluating the role of CSF lactate in the differential diagnosis between acute bacterial and aseptic meningitis. The authors concluded that CSF lactate is a good single indicator and a better marker compared with conventional markers. But concerns remain because of poor quality of included studies, lack of proper 'gold standard', and limited applicability. More studies with a rigorous design are needed to determine definitively whether CSF lactate assay is a reliable and valid marker to distinguish between acute bacterial meningitis and aseptic meningitis.
\end{abstract}

Distinguishing bacterial from viral meningitis/aseptic meningitis (VM/AM) is an age-old problem. The distinction is important because bacterial meningitis (BM) requires urgent intravenous antibiotic administration in the hospital whereas AM is self-limiting. A reliable and valid marker is necessary to make this distinction.

In the previous issue of Critical Care, Huy and colleagues [1] summarize data from 25 studies describing the test characteristics of cerebrospinal fluid (CSF) lactate in the differential diagnosis between acute BM and AM. The methodology used by the authors is reasonably sound and inspires confidence in the results. Reviewer bias was controlled with the use of two

*Correspondence: drkameshwarprasad@yahoo.co.in Department of Neurology, All India Institute of Medical Sciences, Ansari Nagar, New Delhi-110029, India independent reviewers for selection of studies, data extraction, and quality assessment. The chance-corrected agreement (kappa $=0.898$ ) between the reviewers was very high for study selection, although the same was not reported for data extraction and quality assessment.

Huy and colleagues used well-established criteria to assess the quality of the selected studies, and examined and explored heterogeneity using standard methods and summarized the results using a summary receiver operator characteristic (SROC) curve. The authors presented $Q$ value and area under the curve (AUC) as measures of accuracy. The AUC of CSF lactate concentration was 0.9840 , indicating excellent accuracy. The authors concluded that CSF lactate is a good single indicator to distinguish BM from AM and a better marker than other conventional markers. However, comments on threats to internal validity and generalizability of the findings are warranted.

\section{Threats to internal validity}

The quality of a meta-analysis can be only as good as the included studies ('GIGO' principle: garbage in, garbage out). The quality of studies included in the review by Huy and colleagues is somewhat unsettling. Specifically, reported blinding of lactate assay in only 3 (13\%) studies and consecutive or random recruitment of participants in $12(50 \%)$ is a matter of concern. It is possible that other studies blinded the lactate assay without reporting the fact, but this matter remains speculative in the absence of confirmation from the study authors. Compromised quality of original studies threatens the validity of the conclusions.

Lack of a proper 'gold standard' for AM or VM is a vexing problem in this area of research. When an imperfect standard is used to evaluate a diagnostic test, distortions occur in the commonly used measures of test performance, like sensitivity/specificity [2]. Distorted measures carry the error in their meta-analysis. This review suffers from this error. 
Two comments on the comparison of CSF lactate assay with conventional CSF markers are warranted. First, clinicians diagnose AM on the basis of a pattern of a combination of findings on conventional CSF markers (CSF total number of leukocytes, CSF glucose, CSF/ plasma glucose quotient, and CSF protein), not on individual markers. Thus, it is clinically relevant to compare this pattern and the CSF lactate assay and to determine whether the assay adds significantly to the pattern. However, the authors did not address this question; this was probably because they did not have access to the individual patient data that are necessary to perform this comparison. The review, therefore, fails to answer this question.

Second, the authors assert a lower accuracy of individual CSF markers compared with the CSF lactate test based on point estimates of AUC. It is not clear whether the observed differences in AUC are statistically significant. An objective assessment of this is possible by using the Hanley test [3] and by calculating the confidence interval around the estimates.

\section{Applicability}

CSF lactate assay is not available in most centers in developing countries and rural settings. This and the fact that many patients receive antibiotics before lumbar puncture compromise the applicability of the findings.

\section{Summary}

The review is a worthwhile contribution to the field, has a sound methodology, and provides a summary of the results from published data. Clearly, a meta-analysis of individual patient data and more studies are required to determine definitively whether CSF lactate assay is a reliable and valid marker to distinguish between BM and AM.

\section{Abbreviations}

AM, aseptic meningitis; AUC, area under the curve; BM, bacterial meningitis; CSF, cerebrospinal fluid; VM, viral meningitis.

\section{Competing interests}

The authors declare that they have no competing interests.

Published: 14 January 2011

References

1. Huy TN, Thao NTH, Diep DTN, Kikuchi M, Zamora J, Hirayama K: Cerebrospinal fluid lactate concentration to distinguish bacterial from aseptic meningitis: a systemic review and meta-analysis. Crit Care 2010, 14:R240.

2. Valenstein PN: Evaluating diagnostic tests with imperfect standards. Am J Clin Pathol 1990, 93:252-258.

3. Hanley JA, MCNeil BJ: A method of comparing the areas under receiver operating characteristic curves derived from the same cases. Radiology 1983, 148:839-843.

doi:10.1186/cc9396

Cite this article as: Prasad K, Sahu JK: Cerebrospinal fluid lactate: Is it a

reliable and valid marker to distinguish between acute bacterial meningitis and aseptic meningitis? Critical Care 2011, 15:104. 